\title{
(6) OPEN ACCESS \\ Bilateral adaptive deep brain stimulation is effective in Parkinson's disease
}

\author{
Simon Little, ${ }^{1}$ Martijn Beudel, ${ }^{1,2}$ Ludvic Zrinzo, ${ }^{3}$ Thomas Foltynie, ${ }^{3}$ Patricia Limousin, ${ }^{3}$ \\ Marwan Hariz, ${ }^{3}$ Spencer Neal, ${ }^{3}$ Binith Cheeran, ${ }^{1}$ Hayriye Cagnan, ${ }^{1}$ James Gratwicke, ${ }^{3}$ \\ Tipu Z Aziz, ${ }^{1,4}$ Alex Pogosyan,, ${ }^{1,5}$ Peter Brown ${ }^{1,5}$
}

\begin{abstract}
${ }^{1}$ Nuffield Department of Clinical Neurosciences, John Radcliffe Hospital, University of Oxford, Oxford, UK

${ }^{2}$ Department of Neurology, University Medical Centre Groningen, University of Groningen, Groningen, The Netherlands

${ }^{3}$ Unit of Functional Neurosurgery, Sobell Department of Motor Neuroscience \& Movement Disorders, UCL Institute of Neurology, London, UK ${ }^{4}$ Nuffield Department of Surgical Sciences, John Radcliffe Hospital, University of Oxford, Oxford, UK ${ }^{5}$ The Medical Research Council Brain Networks Dynamics Unit, University of Oxford, Oxford, UK
\end{abstract}

\section{Correspondence to} Professor Peter Brown, The Medical Research Council Brain Networks Dynamics Unit at the University of Oxford, Oxford OX1 3TH, UK;

peter.brown@ndcn.ox.ac.uk

$S L$ and $M B$ are joint first authors.

Received 2 April 2015 Revised 13 August 2015 Accepted 17 August 2015 Published Online First 30 September 2015
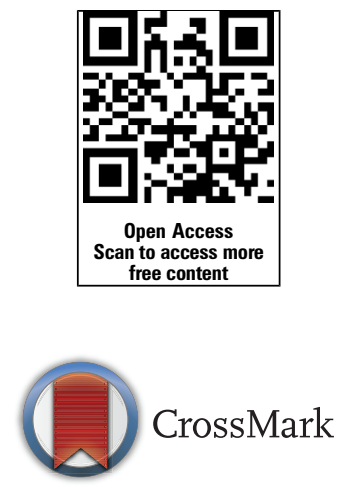

To cite: Little $S$, Beudel M, Zrinzo L, et al. J Neurol Neurosurg Psychiatry 2016;87:717-721.

\section{ABSTRACT}

Introduction \& objectives Adaptive deep brain stimulation (aDBS) uses feedback from brain signals to guide stimulation. A recent acute trial of unilateral aDBS showed that aDBS can lead to substantial improvements in contralateral hemibody Unified Parkinson's Disease Rating Scale (UPDRS) motor scores and may be superior to conventional continuous DBS in Parkinson's disease (PD). We test whether potential benefits are retained with bilateral $\mathrm{aDBS}$ and in the face of concurrent medication.

Methods We applied bilateral aDBS in 4 patients with PD undergoing DBS of the subthalamic nucleus. aDBS was delivered bilaterally with independent triggering of stimulation according to the amplitude of $\beta$ activity at the corresponding electrode. Mean stimulation voltage was $3.0 \pm 0.1$ volts. Motor assessments consisted of double-blinded video-taped motor UPDRS scores that included both limb and axial features.

Results UPDRS scores were $43 \%$ ( $p=0.04$; Cohen's $d=1.62$ ) better with aDBS than without stimulation. Motor improvement with aDBS occurred despite an average time on stimulation (ToS) of only $45 \%$. Levodopa was well tolerated during aDBS and led to further reductions in ToS.

Conclusion Bilateral aDBS can improve both axial and limb symptoms and can track the need for stimulation across drug states.

\section{INTRODUCTION}

Deep brain stimulation (DBS) is an established treatment for advanced Parkinson's disease (PD). ${ }^{1}$ However, its applicability is limited by costs, side effects and partial efficacy. ${ }^{2}$ That symptoms fluctuate based on cognitive/motor load, stress and medication status in PD is well established. ${ }^{3}$ Increasing evidence suggests that these fluctuations are associated with varying levels of subcortical $\beta$ (13$30 \mathrm{~Hz}$ ) oscillations, changes in the amplitude of which correlate with motor improvement in response to treatment. ${ }^{4-7}$ Building on a pioneering adaptive DBS (aDBS) study in the parkinsonian non-human primate, ${ }^{8}$ we recently successfully applied aDBS to patients with PD by triggering stimulation off the amplitude of $\beta$ activity in the local field potential (LFP) recorded from the subthalamic nucleus (STN). ${ }^{9}$ This study showed that aDBS was more effective than continuous DBS (cDBS) despite $<50 \%$ of the total time on stimulation (ToS). However, aDBS was applied unilaterally, with only 5 min stimulation and no assessment of axial symptoms nor control in different drug states.

Bilateral stimulation is essential if aDBS is to be progressed towards clinical applicability. However, this presents technical challenges. Although the subcortical $\beta$ network is significantly coherent bilaterally, ${ }^{10}$ levels of amplitude co-modulation and phase synchronisation between STNs are low. ${ }^{11}$ This suggests that bilateral aDBS may require independent sensing and stimulating. We tested the efficacy of bilateral aDBS in PD, using independent bilateral sensing and stimulation, and in two patients we assessed how aDBS responded to levodopa administration.

\section{PATIENTS AND METHODS}

We tested four male patients with advanced idiopathic PD undergoing DBS of the STN (table 1). All patients gave their informed written consent (approved by the local ethics committee). Patients underwent surgery in a two-stage procedure with bilateral quadripolar Medtronic (3389) electrode placement and stimulator implantation separated by 1 week, as previously described. ${ }^{9}{ }^{12}$ All testing was performed 2-6 days after electrode implantation.

We recorded bipolar LFP activity from the STN electrode contacts after overnight withdrawal of anti-parkinsonian medication. Recordings were made using a band-pass $(3-37 \mathrm{~Hz})$ amplifier that has been previously described. ${ }^{9}{ }^{13}$ However, in this experiment, the amplifier and stimulator independently served both hemispheres and was miniaturised, fixed to the patient on their belt and connected to the base unit by a $10 \mathrm{~m}$ cable allowing assessment of ambulation and axial signs.

Bipolar recordings were made from the contact pair $(0-2$ or $1-3)$ exhibiting the greatest $\beta$ amplitude determined for each hemisphere in the unstimulated and OFF-medication state. We then determined a single-centre frequency and bandwidth which encompassed both of these peaks and set this for our second stage of filtering (table 1). Online $\beta$ amplitudes were obtained by rectifying and smoothing ( $400 \mathrm{~ms}$ moving average) the filtered LFP for each hemisphere. $\beta$ Amplitude was then used to control triggering of stimulation via a user-defined threshold through a portable computer for each side independently. ${ }^{9}$ Stimulation was delivered by a dual version of our previously described stimulator. ${ }^{9}$ On triggering, stimulation was sustained until $\beta$ amplitude fell below threshold 
Table 1 Clinical and stimulation details of patients

\begin{tabular}{|c|c|c|c|c|c|}
\hline & Patient 1 & Patient 2 & Patient 3 & Patient 4 & Mean+SEM \\
\hline Age (years) & 66 & 41 & 52 & 54 & $53.3 \pm 5.1$ \\
\hline Disease duration (years) & 6 & 7 & 8 & 25 & $11.5 \pm 4.5$ \\
\hline Preoperative UPDRS off-drugs & 49 & 50 & 40 & 32 & $42.8 \pm 4.2$ \\
\hline Preoperative UPDRS on-drugs & 19 & 21 & 11 & 4 & $13.8 \pm 3.9$ \\
\hline Surgery location & Oxford & London & London & London & \\
\hline Initial symptom & Tremor & Bradykinesia & Tremor & Rigidity & \\
\hline Primary DBS indication & Refractory tremor & ON-OFF fluctuations & Severe OFF states & OFF-related dystonia & \\
\hline Preoperative drugs (mg/day) & $\begin{array}{l}\text { Levodopa } 1000 \\
\text { Pramipexole } 3.15\end{array}$ & $\begin{array}{l}\text { Levodopa } 625 \\
\text { Amantadine } 100\end{array}$ & Levodopa 500 Rotigotine 4 & $\begin{array}{l}\text { Levodopa } 1600 \\
\text { Pramipexole } 0.375 \\
\text { Tolcapone } 800\end{array}$ & \\
\hline Online digital filter range $(\mathrm{Hz})$ & $20 \pm 3$ & $21 \pm 3$ & $25 \pm 3$ & $20 \pm 6$ & $21.5 \pm 0.8$ \\
\hline Stimulation voltage (V, L/R) & $3.4 / 3.4$ & $3.4 / 3.3$ & $2.5 / 2.4$ & $3.0 / 3.0$ & $3.0 \pm 0.1$ \\
\hline aDBS ToS (\%, L/R) & $25 / 38$ & $53 / 52$ & $35 / 36$ & $62 / 60$ & $45.0 \pm 4.8$ \\
\hline
\end{tabular}

aDBS, adaptive deep brain stimulation; L, left; R, right; SEM, SE of the mean; ToS, time on stimulation; UPDRS, Unified Parkinson's Disease Rating Scale Part III (motor).

(figure 1). Stimulation pulses were charge-balanced, monopolar and symmetrically biphasic $(130 \mathrm{~Hz}$, pulse width $100 \mu \mathrm{s}$, anodal pulse first then a $20 \mu$ s delay before the balancing cathodal pulse). An impedance of $0.5 \mathrm{k} \Omega$ was assumed for calculation of total electrical energy.

The stimulation voltage was determined during aDBS. The voltage was adjusted, along with the $\beta$ amplitude trigger threshold, aiming for clinical benefit without paraesthesia, and a total ToS of approximately 50\%..$^{9}$ The contact selected for stimulation was that which lay between the contacts used for recording. ${ }^{13}$ Stimulation begun at $0.5 \mathrm{~V}$ and was increased by $0.1 \mathrm{~V}$ increments in order to reach a clinically effective voltage as determined by repeat assessments of rigidity and finger tapping by the attending (unblinded) neurologist. This voltage was then taken forward for formal testing and blinded assessment.

Voltage testing was performed separately, leading to two independent stimulation voltages (table 1). Patients were clinically assessed during aDBS and no stimulation. The order of experimental conditions was randomised. We compromised on a 5 min washout period between conditions so as to ensure the acceptability of the experimental session which then lasted about $1.5 \mathrm{~h}$ in two patients and $2.5 \mathrm{~h}$ in those two patients that went on to be stimulated during a levodopa treatment cycle. Stimulation (or no stimulation) was given for $15 \mathrm{~min}$ and clinical testing performed thereafter. In between experimental conditions, we tested the patients to confirm return to baseline before the next condition as judged through finger tapping.

Our primary outcome measure was the motor Unified Parkinson's Disease Rating Scale Part III (UPDRS III) ${ }^{14}$ with aDBS compared with no stimulation. Our secondary outcomes were power saving expressed as mean total electrical energy delivered with aDBS compared with similar, hypothetical, continuous stimulation and the correlation between $\beta$ power and ToS. The patients were blinded by being kept unaware of the stimulation conditions throughout the experiment. In particular, the patients did not experience paraesthesia during aDBS.

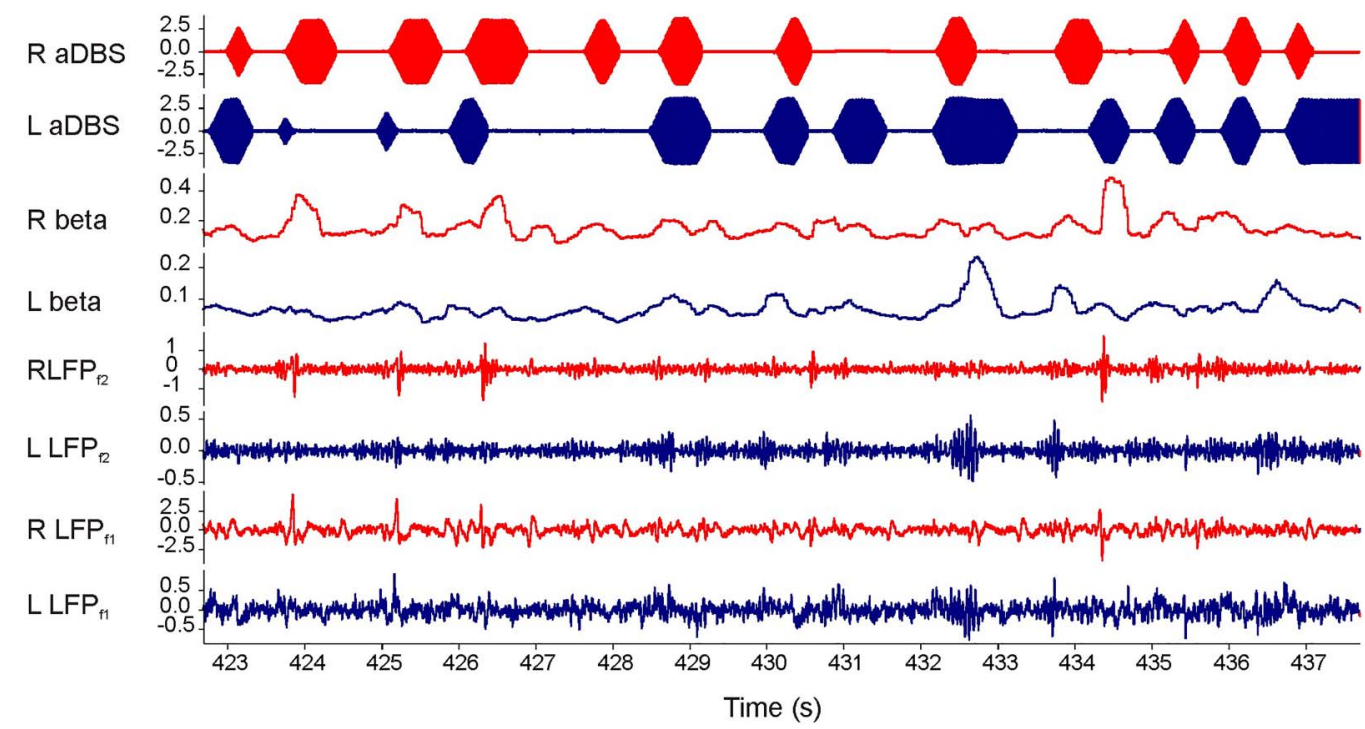

Figure 1 Screen shot of $15 \mathrm{~s}$ bilateral adaptive deep brain stimulation (aDBS) in patient 1. Bottom two traces are local field potentials (LFPs) after first stage analogue filtering (LFPf1; 3-37 Hz). Third and fourth traces from bottom show LFPs after second stage digital filtering around patient specific $\beta$ peak (LFPf2; $20 \pm 3 \mathrm{~Hz}$ ). The two traces above are the online readouts of the filtered $\beta$ amplitude after rectification, smoothing and thresholding. The top two traces show bursts of ramped stimulation in response to $\beta$ amplitude threshold crossing. Blue and red traces are from left and right electrodes, respectively. Note stimulation across the two sides is discontinuous and independent. 
Although patients do sometimes experience paraesthesia during conventional DBS, the latter delivers more than twice the electrical energy used here. Bursts of stimulation during the aDBS practised here were short-lived, ramped in onset and offset and, on average, interspersed by periods of non-stimulation for 55\% of the time (see table 1). UPDRS III assessments were also blinded by videoing them and having the videos rated (rigidity items excluded) by two external movement disorders specialists who were not involved in the experiment and were also unaware of the experimental conditions. There was strong concordance between the two raters with only an 11\% average absolute difference between matched scores $(p=0.85)$. UPDRS III scores were further divided into limb bradykinesia (items 817) and axial symptoms (arise from chair (18), gait (19), stability (21), posture (22) and overall bradykinesia (23)). Unblinded rigidity assessments were also reported separately for both experimental conditions.

Clinical data are described using means and SE of means, were normally distributed (Kolmogorov-Smirnov test, $p>0.05$ ) and analysed using two-sided $t$ tests ( $t$ statistics with degrees of freedom shown along with $\mathrm{p}$ values). The first part of each recording during which the stimulation threshold was heuristically determined and any periods with marked electrical artefacts were excluded. We analysed the first (concatenated if necessary) section of $10 \mathrm{~min}$ artefact-free data after a stable threshold was obtained in each participant. Spectral analysis was conducted using Welch's method with a Hamming window (1 s). $\beta$ Power and ToS were averaged over non-overlapping $10 \mathrm{~s}$ blocks and normalised to the average value of the first minute. To analyse the within-participant relation between mean $\beta$ power per $10 \mathrm{~s}$ and total time on aDBS, between ToS per $10 \mathrm{~s}$ and total time on $\mathrm{aDBS}$, and between ToS and $\beta$ power per $10 \mathrm{~s}$ Spearman's correlations were estimated. Correlation coefficients were Fisher transformed, averaged and back-transformed. In patients 3 and 4 , we performed additional, prolonged recordings of 45 and 60 min duration, respectively. At the beginning of these recordings, $100 \mathrm{mg}$ levodopa was administered and the patients reported a transition from a clinical OFF to ON state which was confirmed by clinical examination of rigidity and bradykinesia. We performed change-point analysis to objectively identify when there was a simultaneous change in the ToS. Change-point analysis iteratively uses a combination of timevarying cumulative sum charts and bootstrapping to detect changes in time series. ${ }^{15}$ To avoid violation of the independent errors assumption, ToS was averaged over successive groups of three data points (where each data point was the average ToS over $10 \mathrm{~s}$ ). Ten thousand bootstraps were performed in each test and only changes with probabilities of $>99 \%$ shown. Significant changes in signal were depicted by means of different confidence limits. In one of the two patients (patient 4) a formal UPDRS III was performed, videoed and blindly rated during the levodopa challenge.

\section{RESULTS}

\section{Clinical effect}

The mean UPDRS part III score OFF medication without stimulation in the days following surgery was $33.5 \pm 5.4$ in the four patients. Individual and group data are illustrated in figure 2. There was a substantial improvement with aDBS in every participant ( $43 \%$ reduction: $\mathrm{t}_{3}=3.24, \mathrm{p}=0.04$; Cohen's $\mathrm{d}=1.62$ ). For limb bradykinesia and axial symptoms, the reductions were $37 \pm 10 \%$ and $39 \pm 5 \%$, respectively. Remaining items, including tremor, speech, facial expression and freezing, improved by

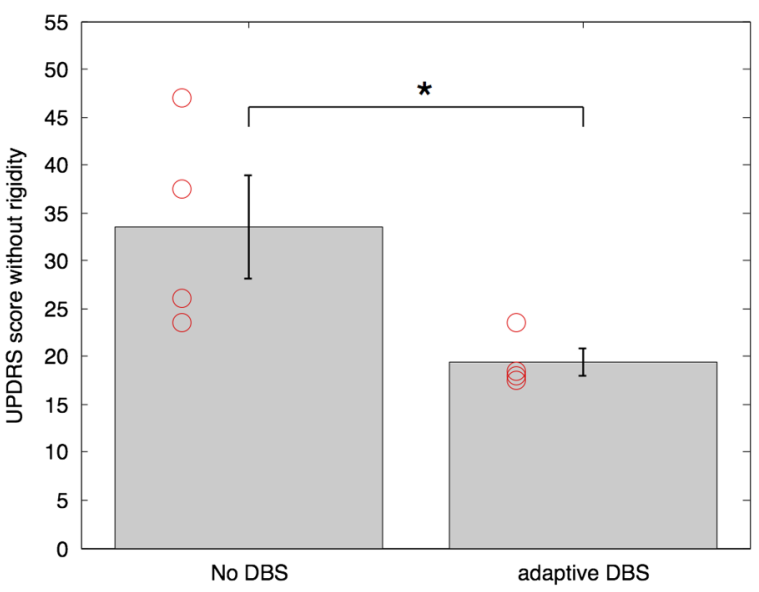

Figure 2 Group mean blinded UPDRS motor scores in the two experimental conditions and their SEs. *Indicates significance with $p<0.05$. Red circles depict individual data. DBS, deep brain stimulation; UPDRS, Unified Parkinson's Disease Rating Scale.

$55 \pm 12 \%$. Unblinded rigidity scores were $9.5 \pm 1.5$ in the condition without stimulation and $6.4 \pm 0.55$ in the aDBS condition.

\section{Power savings}

Total ToS for the whole stimulation period of aDBS was $45 \pm 4.8 \%$ averaged over the eight sides (4 subjects). Furthermore, LFP $\beta$ amplitude and ToS tended to drop during the first $10 \mathrm{~min}$. This was evidenced by the negative correlation between mean $\beta$ power and total time on aDBS, and mean ToS and total time on aDBS across the eight sides. For $\beta$, the average Spearman's correlation coefficient was $-0.23 \pm 0.08$ (single sample $t$ test, $p=0.02)$. For ToS, the average Spearman's correlation coefficient was $-0.20 \pm 0.15(\mathrm{p}=0.21)$. The mean total electrical energy delivered with aDBS was $223 \pm 31 \mu \mathrm{W}$. Conventional continuous stimulation at the same voltage would have delivered $491 \pm 44 \mu \mathrm{W}$.

\section{Response to levodopa}

A prolonged recording following administration of levodopa medication in patient 3 resulted in a self-reported clinical effect $30 \mathrm{~min}$ after ingestion when the average ToS also significantly dropped (figure 3). In the prolonged recording in patient 4 , a clinical effect was seen $40 \mathrm{~min}$ after levodopa. Again, the average ToS significantly dropped at this point (figure 3). Importantly, this drop in ToS occurred without deterioration in motor state, which instead improved from a blinded video-rated UPDRS III score of 17.5 with aDBS OFF drug, to 6.5 on aDBS and levodopa. The blinded video rating of items $1-8$ of the Abnormal Involuntary Movement Scale was 1 out of 50. Note that on both sides of the two patients, change-point analysis of the ToS per $10 \mathrm{~s}$ was able to independently identify when patients turned $\mathrm{ON}$ after medication. Change point analysis of ToS (figure 3) also suggested that the progressive drop in $\beta$ power and ToS seen at the very outset of aDBS plateaued with continued aDBS.

\section{Beta tracking}

$\beta$ Power showed a positive correlation with ToS in each participant in each hemisphere. The average Spearman's correlation coefficient was $0.62 \pm 0.12$. In $7 / 8$ hemispheres, this correlation was significant. In the recordings $\mathrm{ON}$ and $\mathrm{OFF}$ drugs in patients 3 and 4 (4 hemispheres) similar, even 
Figure 3 Per cent ToS during prolonged aDBS after levodopa administration at time 0 and its dependency on $\beta$ power. (A) Grey blocks indicate the periods of stable ToS as identified by change-point analysis $(p<0.01)$. The vertical extent of the blocks denotes the confidence limits of blocks between significant change points and these blocks are centred on the mean of the stable period. The vertical arrows denote when clinical improvement due to levodopa was first manifest. ToS dropped at the onset of the clinical effect of levodopa. Note that prior to this, ToS was stable for about $30 \mathrm{~min}$ or more, suggesting that the progressive drop in ToS at the very outset of aDBS plateaued with continued aDBS. (B) Scatter plots of $\beta$ power and ToS per $10 \mathrm{~s}$ block. Grey line is the product of linear regression. aDBS, adaptive deep brain stimulation; STN, subthalamic nucleus; ToS, time on stimulation.
A
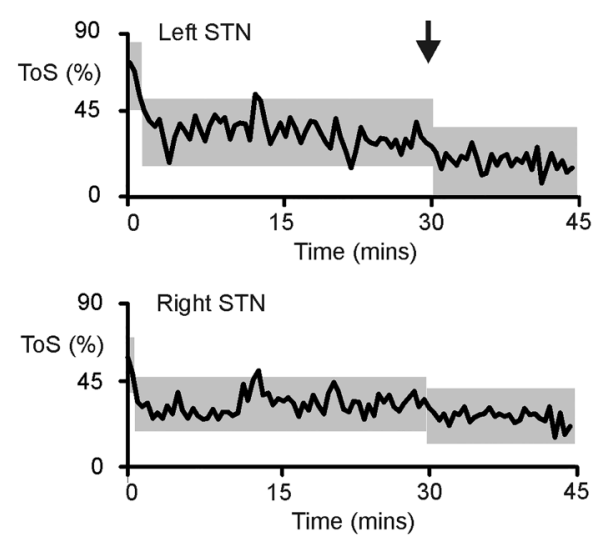

B

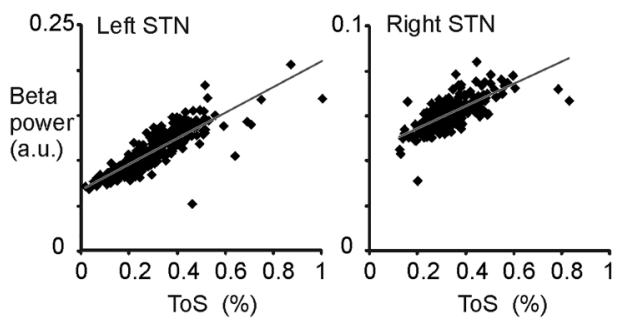

Case 4
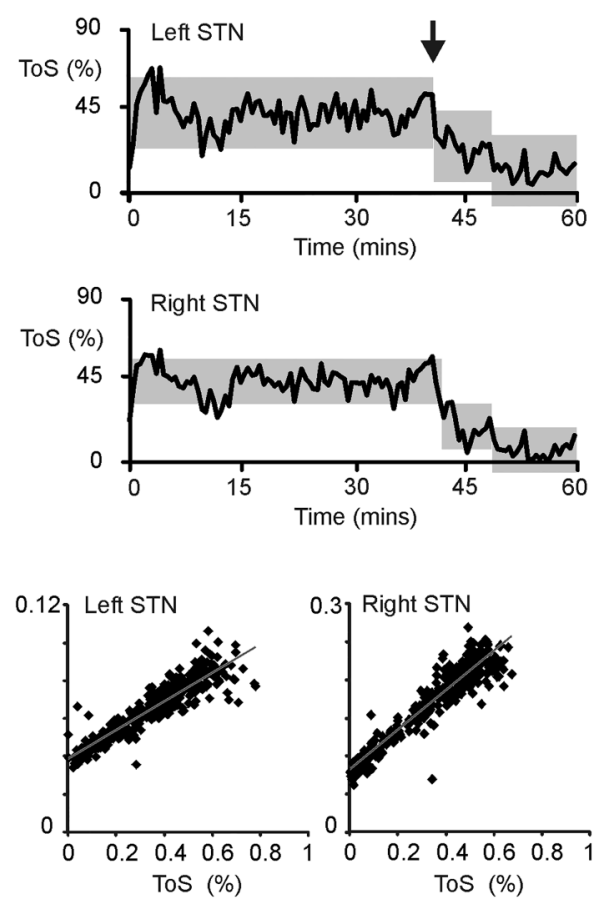

stronger, correlations were present. The average Spearman's correlation coefficient for these recordings was $0.884 \pm 0.147$ (4 hemispheres).

\section{DISCUSSION}

We have previously shown that acute unilateral aDBS improves limb function more than unilateral cDBS. ${ }^{9}$ We demonstrate that bilateral aDBS using two autonomous sensing-and-stimulate systems is well tolerated and improves both axial and limb motor function. The mean effect size was a $43 \%$ reduction in blinded UPDRS scores relative to no stimulation and this was substantial with respect to the variability in the sample, as determined by Cohen's d. This large effect size of aDBS enabled statistically significant conclusions even though our sample was small. Moreover, we found that aDBS was appropriately modulated by levodopa administration, with a further reduction in stimulation, potentially preventing excessive combined therapy and dyskinesias. These findings suggest that bilateral aDBS may prove a safe, effective and highly efficient form of DBS in PD.

The scale of improvement with aDBS was striking, even though stimulation was only on for $45 \%$ of the time, averaged across the eight hemispheres. The latter is of critical importance as it impacts on both side effects and the life time of implanted battery systems. The $43 \%$ improvement with aDBS was similar to that seen with unilateral aDBS, ${ }^{9}$ and similar to that achieved with cDBS, when this has been similarly assessed through blinded rating of videos in other studies. ${ }^{16-18}$ Such reports tend to afford lower effect sizes than unblinded assessments, with blinded cDBS improvement estimates ranging between $25 \%$ and $38 \%$ for assessments of chronic bilateral stimulation with implanted systems ${ }^{16-18}$ and $31 \%$ with acute postoperative unilateral cDBS. ${ }^{9}$

Several experimental limitations may have biased against the detection of all but major effect sizes. Our sample size was small and stimulation regimes were tested only a few days after electrode implantation, when stun effects are present leading to increased electrode impedances and temporary improvement in baseline impairments. ${ }^{19-21}$ The presence of a stun effect in our sample was evidenced by the difference between the mean OFF-medication OFF-stimulation UPDRS score in the days following surgery $(33.5 \pm 5.4$, see Results section) and that recorded preoperatively $(42.8 \pm 4.2$, see table 1$)$. We were also restricted to stimulating one of the middle two contacts of the implanted electrode on each side. Finally, there may have been incomplete washout between conditions. Although the randomised presentation of conditions should have ameliorated this in so far as incomplete washout should apply to both conditions, this cannot be relied on with a small sample, such as in this study, where order effects may still be influential. Furthermore, although the evidence suggests that the effects of conventional cDBS at least, are substantially, but not completely diminished after $5 \mathrm{~min}$ of washout, as used here, ${ }^{22-24}$ the time course of washout for axial symptoms is longer and the time course of the washout of aDBS is unknown. However, longer washout periods would have risked making the experiment unacceptably long for the immediate postoperative period. Nevertheless, these different factors were insufficient to obfuscate the large and significant effect size of aDBS.

The possibility that bilateral aDBS may be effective despite a mean ToS of $45 \%$ (with a comparable reduction in energy consumption) may prove to have major advantages in limiting stimulation side effects. Theoretical and empirical evidence suggests that conventional DBS can involve direct spread to local fibre tracts and interfere with physiological network activity and thereby compromise motor skills, including speech. ${ }^{25}$ Although current spread to fibre tracts will still occur with aDBS, overall side effects may potentially lessen as stimulation is more limited in time and delivered only when $\beta$ network activity is pathologically synchronised. ${ }^{26}$ This potential for side effect reduction is underscored by the response of the stimulation system to levodopa administration. Stimulation on time was significantly reduced, in response to $\beta$ suppression with levodopa. ${ }^{4}$

Overall, this study demonstrates that bilateral aDBS is well tolerated, improves total motor UPDRS III scores, and yet requires on average $45 \%$ of the standard ToS. The large effect size of aDBS enabled statistically robust conclusions even though our sample 
was small. Since $\beta$ LFP activity is a relatively consistent feature of the STN in $\mathrm{PD}^{2}$ and consistent over long periods of time, ${ }^{21}{ }^{27}$ this approach has the potential to be realised clinically. However, much work remains to be done to explore and optimise the full range of parameters and algorithms that could be employed. Hereafter, the next step will be a head-to-head comparison of aDBS with standard continuous high-frequency DBS, with voltages and other stimulation parameters independently optimised for each stimulation regime in a large patient cohort.

Acknowledgements This work was funded by the Medical Research Council, Rosetrees Trust and National Institute for Health Research (NIHR) Oxford Biomedical Research Centre. The UCL Unit of Functional Neurosurgery is supported by The Parkinsons Appeal UK, and the Monument trust. The work was partly carried out in the NIHR Cognitive Health Clinical Research Facility, Oxford.

Contributors $\mathrm{SL}$ and $\mathrm{MB}$ conducted the experiments, analysed the data and wrote the first draft of the manuscript. JG assisted with the experiments. LZ, TF, PL, MH, $B C$ and TZA were involved in the patient selection, operation and postoperative management. SN, $\mathrm{HC}$ and $\mathrm{AP}$ were responsible for the development of the stimulation device. PB initiated the study. All authors have contributed to the critical evaluation of the manuscript in its current form.

Competing interests None declared.

Ethics approval National Research Ethics Service Committee South Central, Oxford.

Provenance and peer review Not commissioned; externally peer reviewed.

Open Access This is an Open Access article distributed in accordance with the terms of the Creative Commons Attribution (CC BY 4.0) license, which permits others to distribute, remix, adapt and build upon this work, for commercial use, provided the original work is properly cited. See: http://creativecommons.org/ licenses/by/4.0/

\section{REFERENCES}

1 Deuschl GL, Schade-Brittinger C, Krack P, et al. A randomized trial of deep-brain stimulation for Parkinson's disease. N Engl J Med 2006;31:896-908.

2 Deuschl G, Paschen S, Witt K. Clinical outcome of deep brain stimulation for Parkinson's disease. Handbook of clinical neurology; brain stimulation. Amsterdam: Elsevier, 2013

3 Pradhan SD, Brewer BR, Carvell GE, et al. Assessment of fine motor control in individuals with Parkinson's disease using force tracking with a secondary cognitive task. J Neurol Phys Ther 20101;34:32-40.

4 Kühn AA, Kupsch A, Schneider GH, et al. Reduction in subthalamic 8-35Hz oscillatory activity correlates with clinical improvement in Parkinson's disease. Eur $J$ Neurosci 2006;23:1956-60.

5 Ray NJ, Jenkinson N, Wang S, et al. Local field potential beta activity in the subthalamic nucleus of patients with Parkinson's disease is associated with improvements in bradykinesia after dopamine and deep brain stimulation. Exp Neurol 2008;213:108-13.

6 Weinberger M, Mahant N, Hutchison WD, et al. Beta oscillatory activity in the subthalamic nucleus and its relation to dopaminergic response in Parkinson's disease. J Neurophysiol 2006;96:3248-56.
7 Kühn AA, Tsui A, Aziz T, et al. Pathological synchronisation in the subthalamic nucleus of patients with Parkinson's disease relates to both bradykinesia and rigidity. Exp Neurol 2009;215:380-7.

8 Rosin B, Slovik M, Mitelman R, et al. Closed-loop deep brain stimulation is superior in ameliorating parkinsonism. Neuron 2011;72:370-84.

9 Little S, Pogosyan A, Neal S, et al. Adaptive deep brain stimulation in advanced Parkinson disease. Ann Neurol 2013;74:449-57.

10 de Solages C, Hill BC, Koop MM, et al. Bilateral symmetry and coherence of subthalamic nuclei beta band activity in Parkinson's disease. Exp Neurol 2010;221:260-6.

11 Little S, Tan H, Anzak A, et al. Bilateral functional connectivity of the basal ganglia in patients with Parkinson's disease and its modulation by dopaminergic treatment. PLOS ONE 2013:8:e82762

12 Foltynie T, Hariz MI. Surgical management of Parkinson's disease. Expert Rev Neurother 2010;10:903-14.

13 Eusebio A, Cagnan H, Brown P. Does suppression of oscillatory synchronisation mediate some of the therapeutic effects of DBS in patients with Parkinson's disease? Front Integr Neurosci 2012;6:47.

14 Goetz CG, Tilley BC, Shaftman SR, et al. Movement Disorder Society-sponsored revision of the Unified Parkinson's Disease Rating Scale (MDS-UPDRS): scale presentation and clinimetric testing results. Mov Disord 2008:23:2129-70.

15 Taylor WA. Change-point analysis: a powerful new tool for detecting changes. 2000. httpwww.variation. comcpatechchangepoint.html

16 Castrioto A, Lozano AM, Poon YY, et al. Ten-year outcome of subthalamic stimulation in Parkinson disease: a blinded evaluation. Arch Neurol Psychiatry 2011;68:1550-6.

17 Ford B, Winfield L, Pullman SL, et al. Subthalamic nucleus stimulation in advanced Parkinson's disease: blinded assessments at one year follow up. J Neurol Neurosurg Psychiatr 2004;75:1255-9.

18 Schuepbach WM, Rau J, Knudsen K, et al. Neurostimulation for Parkinson's disease with early motor complications. N Engl J Med 2013;368:610-22.

19 Lempka SF, Miocinovic S, Johnson MD, et al. In vivo impedance spectroscopy of deep brain stimulation electrodes. J Neural Eng 2009;6:046001.

20 Rosa M, Marceglia S, Servello D, et al. Time dependent subthalamic local field potential changes after DBS surgery in Parkinson's disease. Exp Neurol 2010;222:184-90.

21 Abosch A, Lanctin D, Onaran I, et al. Long-term recordings of local field potentials from implanted deep brain stimulation electrodes. Neurosurgery 2012; 71:804-14.

22 Lopiano L, Torre E, Benedetti F, et al. Temporal changes in movement time during the switch of the stimulators in Parkinson's disease patients treated by subthalamic nucleus stimulation. Eur Neurol 2003;50:94-9.

23 Keresztenyi Z, Valkovic $P$, Eggert $T$, et al. The time course of the return of upper limb bradykinesia after cessation of subthalamic stimulation in Parkinson's disease. Parkinsonism Relat Disord 2007:13:438-42.

24 Cooper SE, Noecker AM, Abboud H, et al. Return of bradykinesia after subthalamic stimulation ceases: relationship to electrode location. Exp Neurol 2011:231: 207-13.

25 Chen CC, Brücke C, Kempf F, et al. Deep brain stimulation of the subthalamic nucleus: a two-edged sword. Curr Biol 2006;16:R952-3.

26 Brittain JS, Sharott A, Brown P. The highs and lows of beta activity in cortico-basal ganglia loops. Eur I Neurosci 2014;39:1951-9.

27 Giannicola G, Rosa M, Servello D, et al. Subthalamic local field potentials after seven-year deep brain stimulation in Parkinson's disease. Exp Neurol 2012;237:312-17. 